\title{
Schizoaffective disorder and psychosis: a reflective case study
}

\author{
Edward Fearnley
}

Ed is a third-year medical student at Lancaster Medical School.

\section{INTRODUCTION}

Schizophrenia, schizoaffective disorder and bipolar disorder represent three of the major psychotic illnesses in psychiatry. Psychosis is a complex state with many causative factors (trauma, drug use and infection) and labyrinthine interactions between genes and the environment. It is life threatening and can even threaten the lives of others. Psychosis may also be 'mood congruent', as witnessed in the grandiose delusions in mania and the nihilistic delusions of depressive illness, and hallucinations can range from the vividly visual, to the auditory, tactile, gustatory and kinetic.

The aetiology and nature of psychosis in mental illness has been a puzzle only partially solved over the past century. The most pertinent point to highlight is that psychosis is not a state that can be universally identified via a biological marker. In certain situations, such as in postoperative delirium, an underlying infective cause may be obvious, but the functional psychotic disorders cannot yet be differentiated in this manner.

This means that psychiatric diagnosis is heavily reliant (although not exclusively) on descriptive psychopathology, meaning that certain constellations of symptoms must be present in the formation of a diagnosis. The enlightened work of Karl Jaspers (1883-1969) and Emil Kraepelin (1856-1926) pioneered this paradigm shift, moving from a chaotically symptomatic 'case-bycase' approach, to an organised, reliable syndromal method of classification. A fundamental schism between schizophrenia and mood disorders consequentially developed. The ICD-I0 (International Classification of Diseases) and DSM-IV (diagnostic and statistical manual of mental disorder) have matured from this.

The work of Sigmund Freud (1856-1939) also popularised the concept of 'neurosis' and 'psychosis' as separate entities. Even as psychoanalytical thought has gradually fallen from vogue, these boundaries are powerfully demarcated in the both the professional and popular collective consciousness.

Over time, however, there has been criticism of this so-called 'Kraepaelin dichotomy', and descriptive psychopathology in general. One diagnosis, however, has the potential to undermine this traditional divide. This is the schizoaffective population; a group displaying symptoms of both schizophrenia and affective illness. Alongside this critique, lies some momentum for an entire paradigm shift in the classification of psychosis (and more broadly, mental illness), from the aforementioned categorical model, to a more valid alternative, such as a spectrum or continuum.

It is in view of these competing viewpoints today that simple case studies retain the ability to highlight the similarities and differences between the psychotic illnesses. They have the potential to contribute to the debate, and the bigger question of whether a paradigm shift would be beneficial for both research and clinical practice.

\section{SCHIZOPHRENIA}

Schizophrenia is a particularly severe form of mental illness. It has a prevalence of $1 \%$ in the general population, reaching $7 \%$ in inner-city areas. UK incidence of schizophrenia is estimated to be between 7-17/100,000 per year. It leads to a 15-year reduction in life expectancy, and in England alone had an estimated cost of $£ 6.7$ billion in 2005. (1) It is an illness that incorporates a broad range of signs and symptoms, a point illustrated by the ICD- 10 classification (see boxes below). ${ }^{2}$

Schizophrenia can also be described in terms of positive, disorganised and negative symptoms. To supplement this, Kurt Schneider (I 887-1967) also developed a list of 'I st rank' symptoms - the presence of being indicative of a schizophrenia diagnosis. Positive symptoms include hallucinations and delusions, negative symptoms refer to a flattening of the affect, apathy and attentional impairment, and disorganised symptoms to thought disorder.

ICD- I 0 diagnostic criteria require at least one very clear symptom from the following list of positive symptoms:

- Thought 'echo', insertion, withdrawal, or broadcasting.

- Delusions of control or influence, clearly referred to body or limb movements, or specific thoughts, actions, or sensations; delusional perception.

- Hallucinatory voices giving a running commentary on the patient's behaviour (2nd person), or discussing him between themselves (3rd person), or other types of hallucinatory voices coming from some part of the body.

- Persistent delusions that are completely impossible (eg being able to control the weather).

Together with two symptoms from a list of less disabling features:

- Persistent hallucinations - auditory, visual, olfactory, accompanied by delusions (which may be fleeting or halfformed) without clear affective content.

- Breaks or interpolations in the train of thought, resulting in incoherence or irrelevant speech or the use of nonsense words.

- Catatonic behaviour, such as excitement, posturing or waxy flexibility, negativism, mutism and stupor.

- Apathy, paucity of speech, and blunting or incongruity of emotional responses unrelated to depression or medication. 
In addition to this, the ICD- 10 subdivides schizophrenia further, into nine different types, ranging from paranoid schizophrenia, to post-schizophrenic depression.

\section{BIPOLAR DISORDER}

Bipolar disorder, historically known as 'manic depression', is characterised by the prominent affective symptoms of mania and depression, with the ability of these mood states to change over time. Lifelong prevalence is estimated to be between $0.3-1.5 \%$, with an annual $\mathrm{NHS}$ cost of $€ 199$ million. ${ }^{(3)}$ Bipolar disorder can be divided into manic, hypomanic and depressive episodes. DSM-IV further divides bipolar disorder in type I and type II, requiring one severe episode for diagnosis. ICD- I0 contrarily requires at least two severe mood episodes, one of which is a manic/hypomanic phase. Psychosis is very common in mania, but is not strictly necessary for diagnosis (psychotic mania can be diagnosed separately). Also, a diagnosis of bipolar disorder may not be immediately evident (it is dependent upon the chronology of the presenting symptoms), thus an initial diagnosis of depression or a manic episode may come first.

\section{SCHIZOAFFECTIVE DISORDER}

Schizoaffective disorder was described in 1933 by Jacob Kasasin (1997-1946). It refers to the exhibition of both prominent affective symptoms and those of schizophrenia, but does not meet the criteria to warrant an exclusive diagnosis in either category. The ICD- 10 criteria require at least a twoweek duration of symptoms before a diagnosis is made and the condition can be further divided into a 'manic' or 'depressive' type, depending on which affective symptoms are prominent.

There are no exact figures of incidence and prevalence of schizoaffective disorder for the UK, although it is thought to be less common than schizophrenia, with an estimated prevalence of I in 200 people. ${ }^{(4)}$

It is in view of this battlefield in mental health today that simple case studies become important for highlighting the similarities and differences between the psychotic illnesses. They have the potential to contribute to the debate, and the bigger question of whether a paradigm shift would be beneficial for both research and clinical practice.

\section{CASE STUDY}

\section{Background}

The patient is a 46-year-old woman, with a current diagnosis of schizoaffective disorder. She has experienced psychotic illness for the past 21 years. The first episode started in the months after her mother committed suicide. Symptoms included a very low mood, becoming socially withdrawn, feeling paranoid, with ideas of reference. Whilst psychotic she attempted suicide and was hospitalised voluntarily for the first time. Over the past two decades she has been in hospital on six occasions, but has not been sectioned under the Mental Health Act. She has attempted suicide on a further four occasions. Over the years, low mood has been a prominent symptom, but third-person auditory hallucinations have also developed over time, becoming the most prominent psychotic symptom at present.

\section{Family history}

Significantly, mental illness exists on both sides of the family. Both her father and uncle were diagnosed with schizophrenia and the mother was diagnosed with manic depression.

\section{Personal history}

There appears to have been no prenatal or obstetric complications, and she reached all speech and motor developmental milestones. Childhood/adolescence was described as disruptive, due partially to parental illness and her developing sexuality. She reached tertiary education and now has a master's degree. Employment opportunities have been limited due to the chronic nature of the illness, although she has been successful carrying out voluntary work. She has been in a happy, longterm relationship for over 15 years. She has no forensic history.

\section{Drug and alcohol history}

She does not drink alcohol, having stopped four years ago for health reasons; she used to drink 50 units a week. She also gave up smoking three years ago to increase the efficacy of the clozapine. There is no history of illicit drugs.

\section{Medication}

Current therapy includes lamotrigine (a mood stabiliser), clozapine (an atypical antipsychotic), and fluoxetine (an SSRI antidepressant). She had previously taken haloperidol and risperidone (typical and atypical antipsychotics); however, they proved ineffective at managing auditory hallucinations. It is this treatment resistance which prompted the use of clozapine. She has no known allergies. However, weight gain, a common side-effect associated with anti-psychotics and anti-depressants, was described.

\section{Medical/surgical history}

The medical history was unremarkable, specifically there was no history of diabetes, thyroid disorders, drug-induced Parkinsonism or epilepsy.

\section{Mental state examination}

On interview, she was appropriately attired, with no signs of self-neglect, displayed a good level of eye contact, and a rapport was established. Speech was of normal content without pressure or poverty and she was not suicidal or delusional, variation, volume, quantity, rate, and rhythm. There was no loosening of associations, flight of ideas, or dysarthria observed. Thought content was not delusional or obsessive, nor did she display any overvalued ideas. Neither did she describe any withdrawal, passivity, broadcasting or insertion of thoughts. Subjectively, she described her mood as well, with no elation, or suicidal intent. Her affect appeared euthymic, with no signs of agitation. No abnormal movements (tics, choreoathetosis) or psychomotor retardation. Perceptually, no illusions or hallucinations, or depersonalisation were described. She was fully conscious, undistracted, orientated in space, time and person; and the interview elicited no long or short-term memory deficiency. She also had full insight into her condition.

\section{CASE DISCUSSION}

This case of schizoaffective disorder highlights several areas of discussion. It illustrates the typical presentation of a patient chronically ill with psychotic illness, and the progression of their illness in a way where the diagnosis changes as the illness 
changes character. The existence of schizoaffective disorder also highlights how it is possible for a patient to have prominent mood symptoms alongside those typically associated with schizophrenia (such as the paranoid delusions or the auditory hallucinations), and also how difficult a diagnosis it can be to make.

Areas to explore include the prognostic value gained from categorical diagnosis, whether new genetic research supports the existence of this existing system, and how management of the patient is affected.

\section{Shared genetics}

Whilst the precise aetiology of functional mental illness is unknown, it is almost certainly multifactorial, with various associations and risk factors already identified. Recent research, including that of genome-wide association studies, has partially illuminated the genetic aetiology of psychotic illness. A familial link has long been known, with the results from twin studies strongly supporting the argument for categorical diagnosis.

\section{Twin/family studies}

Twin studies completed over the years have clearly demonstrated than both conditions carry significant, yet very different, genetic risks. Classically, a review of several twin studies of schizophrenia by Gottsmann and Shields revealed that in both monozygotic and dizygotic twins those with schizophrenia demonstrate a concordance rate of $74 \%$ and $22 \%$ respectively (this was using 'severe schizophrenia', measured by a two-year hospitalisation). More recent studies ascertain these conclusions. ${ }^{(5)}$

Concerning bipolar disorder as well, an individual has a 74\% risk of developing the condition if both parents are affected, and a $27 \%$ risk if one parent is ill.(6)

Twin studies and genetic studies in general have been historically useful and widely used in psychiatry, and are some of the clearest evidence yet that schizophrenia and bipolar disorder have independent aetiologies.

However, there have been suggestions that the genetic risk of developing schizophrenia and bipolar disorder overlap by a large amount. A large-scale study of two million families in Sweden (20I I) analysed hospital discharge registers, demonstrating an increased risks of both schizophrenia and bipolar disorder in first-degree relatives of people with either disorder.(7) Genome-wide association studies of over 10,000 people have revealed that the CACNAIC gene expressed in bipolar disorder is also expressed frequently in schizophrenia. ${ }^{(8)}$ Other association studies have discovered other genes associated with both disorders.

These relatively recent insights, particularly shared-gene expression, suggest that psychotic illnesses may not be as discrete a diagnosis as previously thought.

Pertinently, this is also demonstrated in the case study. Whilst it is vital not to submit to 'genetic determinism' and minimise other contributors, such as life events, the family history is significant. The patient had a mother with bipolar disorder and a father with schizophrenia. Whilst only a case study, it does illuminate what is already being described at a population-wide level. Environmental factors cannot be disowned either; 'life events' are known causative factors to illness both independently and as a critical mass of a complex bio-psycho-social web of factors. The family history in the case study further questions this. As well as genetic vulnerability, the death of a parent is a life event in its own right.

In addition, since schizoaffective disorder appears to be a mixture in the phenotypic presentation of schizophrenia and bipolar disorder, there is the possibility that it is a phenotypic mixture of both conditions.

\section{Does schizoaffective disorder 'exist'?}

It is notoriously difficult to weigh up the validity of schizoaffective disorder. There are arguments for its continued inclusion in diagnostic manuals, and arguments for its removal entirely. As a diagnosis, it has been undermined by the fact that the ICD-IO and DSM-IV do not concur on specific diagnostic criteria, whilst DSM-IV requires the delusions and hallucinations to be present for two weeks without affective symptoms). The updated DSM-V, to be published in October 2013, may not even include schizoaffective disorder as a diagnosis. Clearly, as demonstrated by the patient, the symptom cluster is very real and affects many thousands of people. It is as yet unclear whether its removal would benefit these patients, disrupt treatment, or not make much of a difference. Arguments for its removal are largely from those demanding a spectrum approach to psychosis, and consider schizoaffective disorder to be midway along this spectrum, half-way between the traditional end poles of schizophrenia and bipolar disorder.

\section{Are we missing co-morbidity?}

The existence of a patient subgroup experiencing symptoms of both schizophrenia and bipolar disorder could also be demonstrating co-morbidity. Psychiatric co-morbidity is very common (as demonstrated in depression and anxiety), and psychiatric illness is not protective toward the development of other conditions.

The mixed picture of schizophrenia and affective symptoms from the schizoaffective group could simply be describing the group unfortunate enough to develop both schizophrenia and bipolar disorder. The patient from the case study is illustrative of this in the fact that there is a family history of schizophrenia and bipolar disorder (see twin studies described). It is also possible that illness showing a mixed picture of schizophrenia and low mood, may be psychotic major depression. Part of the patient's clinical picture appears to support this diagnosis, such as the persecutory auditory hallucinations and deliberate self harm.

Patients with schizophrenia have an increased risk of developing bipolar disorder and vice versa, therefore it is possible that some patients with a schizoaffective disorder diagnosis may actually suffering from two separate conditions concurrently.

Key differences between schizophrenia and bipolar disorder There are numerous differences existing between psychotic illnesses, which form a strong argument for their continued individuality (see table I below). Both schizophrenia and bipolar do not appear to affect genders unequally; however, rapid-cycling bipolar disorder is more common in females. Schizophrenia, however, presents at a younger age in men (early 20s) and is associated with a poorer prognosis. The entire clinical pattern of schizophrenia is very different too, characterised by a substantial prodromal period, a sharp 
contrast to the often abrupt onset of mania. Bipolar disorder is also commonly described as 'relapsing-remitting', allowing for the patient to achieve a good level of pre-morbid functioning in-between episodes. This is in clear contrast to schizophrenia, however, as each psychotic episode is punctuated by a decline in function, often below that of pre-morbid levels.

The nature of the psychotic experience in the two disorders is also different. Affect is often flat in schizophrenia, something very rare in the momentous mood shifts of bipolar. Whilst not mutually exclusive with bipolar disorder, auditory hallucinations are associated with the diagnostic criteria of schizophrenia. Delusional behaviour in bipolar disorder tends to be mood congruent also, typified with the well-documented grandiose behaviour in mania.

A final, well-documented differentiating factor for the two conditions is the respective suicide rates. Both disorders significantly increase the risk and contribute to the morbidity/mortality of both, yet research shows that between 5-13\% patients with schizophrenia commit suicide. In comparison, bipolar disorder has a suicide rate of 5-20\%. In addition, life expectancy for men with schizophrenia is 18.7 years below the average (78.3 years), in comparison to 13.6 years below the average for men with bipolar disorder.(9)

\begin{tabular}{|l|l|l|l|l|}
\hline Psychopathology & $\begin{array}{l}\text { Average } \\
\text { age of } \\
\text { onset }\end{array}$ & Male/female & $\begin{array}{l}\text { Prognosis/ } \\
\text { illness } \\
\text { pattern }\end{array}$ & $\begin{array}{l}\text { Pharmacological } \\
\text { treatment }\end{array}$ \\
\hline Schizophrenia & $\begin{array}{l}\text { Late } \\
\text { adolescence/ } \\
\text { early 20s }\end{array}$ & $\begin{array}{l}\text { Equal } \\
\text { prevalence }\end{array}$ & $\begin{array}{l}\text { Poorer } \\
\text { in males, } \\
\text { chronic, } \\
\text { progressive } \\
\text { pattern }\end{array}$ & Anti-psychotics \\
\hline Bipolar disorder & Early/mid 20s & $\begin{array}{l}\text { Equal } \\
\text { prevalence, } \\
\text { higher female } \\
\text { rapid cycling }\end{array}$ & $\begin{array}{l}\text { Relapse/ } \\
\text { remitting } \\
\text { illness }\end{array}$ & $\begin{array}{l}\text { Mood stabilisers } \\
\text { and anti-psychotics }\end{array}$ \\
\hline \multicolumn{3}{|c|}{ Table I Comparison of schizophrenia and bipolar disorder }
\end{tabular}

\section{Different longterm managements}

The longterm management of both conditions suggest a difference in aetiology, or at least that psychosis operates via different mechanisms in different conditions. Fundamentally, it is understood that bipolar disorder is responsive to mood stabilising drugs, and schizophrenia to anti-psychotics. It is also well known that the earlier one starts a pharmacological intervention, the better the prognosis. At face value, this appears to suggest separate aetiologies exist for both conditions. To confound matters, however, anti-psychotics are also mobilised in the treatment of mania. In 1976, Robert Snyder proposed the dopamine hypothesis for schizophrenia, associating the illness with increased levels of dopamine in the brain. This ran alongside research showing that typical anti-psychotics such as chlorpromazine acted at D2 receptors in the mesolimbic pathways, combined with the fact that other potent drugs such as amphetamines and Levodopa can also produce a psychosis. If schizophrenia and bipolar disorder existed on a spectrum of psychosis, it was a reasonable challenge to a shared aetiology.

However, the past 35 years has shown the dopamine hypothesis to be slightly simplistic. Typical anti-psychotics were good at treating the positive symptoms of schizophrenia, but struggled with the negative. Drugs since then, which have proven more successful at treating negative symptoms (and treatment resistance) such as clozapine, are thought to act on D4 and $5 \mathrm{HT}$ receptors as well as D2, prompting a re-evaluation of schizophrenia aetiology. ${ }^{(10)}$ Also, the use of anti-psychotics in the initial acute treatment of mania (or indeed, schizophrenia), does suggest a common mechanism to the symptoms, but does it suggest a common aetiology?

\section{CONCLUSION}

Ideally, the case study has illuminated the diagnostic challenge presented by psychotic illness. The causes of psychosis range widely, from infection, to the unknown. The sheer variation in

\section{A service user's viewpoint about the issues around diagnosis (October 20I2)}

Labelling is a big issue for mental health service users and their families, as society defines the term 'schizophrenic' in a negative context. In terms of identity and identity politics, we service users are at risk of internalising the negative connotations of living with voices and 'psychosis' and the terms 'schizophrenic, 'schizoaffective' or 'bipolar'.

In terms of genetics, which is a contentious issue, my own understanding is based on the stress-vulnerability model. Having both parents who had long-standing mental health issues means that I was vulnerable to mental health issues myself, but the trigger for my first experience of psychosis was the consequence of the intensity of grief following the suicide of my mother. Two further relapses have followed other major life events such as relationship breakdown.

Alongside the use of medication (I take clozapine; lamotrigine and fluoxetine), I have benefitted from working with the concept of psycho-social intervention. This has enabled me to question alternatives to an immediate paranoid reaction to situations and also the fact that we, as service users, have our own understanding of what the voices mean to us and our self-esteem.

Within the service user/carer world there is a debate about the 'recovery model', which challenges both a total focus on the medical model and also the social model. The service user (and where relevant, their carer) becomes the 'expert' of managing their own mental health. This includes identifying 'early warning signs' and when to seek help. In addition, the recovery model includes experiencing and enabling an emotional, social, spiritual and occupational wellbeing and to enjoy a sense of community and neighbourliness not necessarily being symptom free. This is what makes life 'normal'.

In conclusion, I would like to focus on the idea of collaborative working with medical staff, nurses, occupational therapists and social workers both in hospital and in the community working with the concept of holistic care for service users and their families. 
the presentation and clinical outcome of psychotic illness, alongside burgeoning neuro-scientific insights, call for a reassessment of orthodox classifications of discrete categories of psychosis. However, short of a biochemical marker for functional psychosis, the use of symptom clusters to differentiate one group of patients from another is the best tool at our disposal. The formation of a sensible diagnosis of schizophrenia, schizoaffective, or bipolar disorder relies on more than mere symptoms too. As illustrated by the case study, gender, genetics, and the ability of a clinician to take a complete history (establishing the course of the illness) are just as important to the diagnostician. This synergistic concept has never been so pertinent.

A clear diagnosis, however theoretically imperfect, allows for the creation of solid management plans, an estimation of a prognosis and offers an explanation to the patient and carers. It also allows for more reliable research, which with $\mathrm{AMRI}$, molecular biology and further opportunities (unimaginable to Kraepelin) will surely lead to more understanding in the future.

\section{AFTERWORD}

I would like to extend my gratitude to the service user whose life story is described in this case study. The following was written from their own perspective. Thanks also to Dr Linda Ashworth, Nick Mullin and others for their invaluable assistance.

\section{REFERENCES}

I. Mangalore R, Knapp M. Cost of schizophrenia in England. J Ment Health Policy Econ 2007; 10:23-4l

2. World Health Organisation. ICD- 10 Classifications of Mental and Behavioural Disorder: Clinical Descriptions and Diagnostic Guidelines. Geneva:World Health Organisation; 1992

3. Gupta R, Guest F. Annual cost of bipolar disorder to UK society, Br J Psychiatry 2002; 1 80:227-33

4. Castle D. Schizoaffective Disorder. Advances in Psychiatric Treatment. 2012;18:32-3

5, Gotsmann II, Shields ]. A critical review of recent adoption, twin and family studies in schizophrenia. Schizophr Bull |976;2:360-40|

6. Smoller J, Finn, C. Family, twin and adoption studies in bipolar disorder. Am J Med Genet 2003; I 23C(I):48-58

7. Lichtenstein P, Benjamin H, Bjork C. Common genetic determinants of schizophrenia and bipolar disorder in Swedish families: a population based study. Lancet 2009;373:190-1

8. Zhang Q, Shen Q, Xu Z. The effects of CACNAIC gene polymorphism on spatial working memory in both healthy controls and people with schizophrenia or bipolar disorder. Neuropsychopharmacology 20 12;37:677-84

9. Laursen T. Life expectancy among persons with schizophrenia or bipolar affective disorder. Schizophr Res 2011;131:101-4

10. Ananth J, Burgoyne K. How do the atypical antipsychotics work? J Psychiatry Neuroscience 200 1;26:385-94

\section{The Morecambe Bay Medical Journal Prize for the best article by a junior doctor/student}

A prize of $€ 200$ is awarded each year to the author of what is judged to be the best article published in the Journal.

The prize is open to all junior doctors and students. The winner of the 2012 prize will be announced in the Summer 2013 issue of the Journal. 